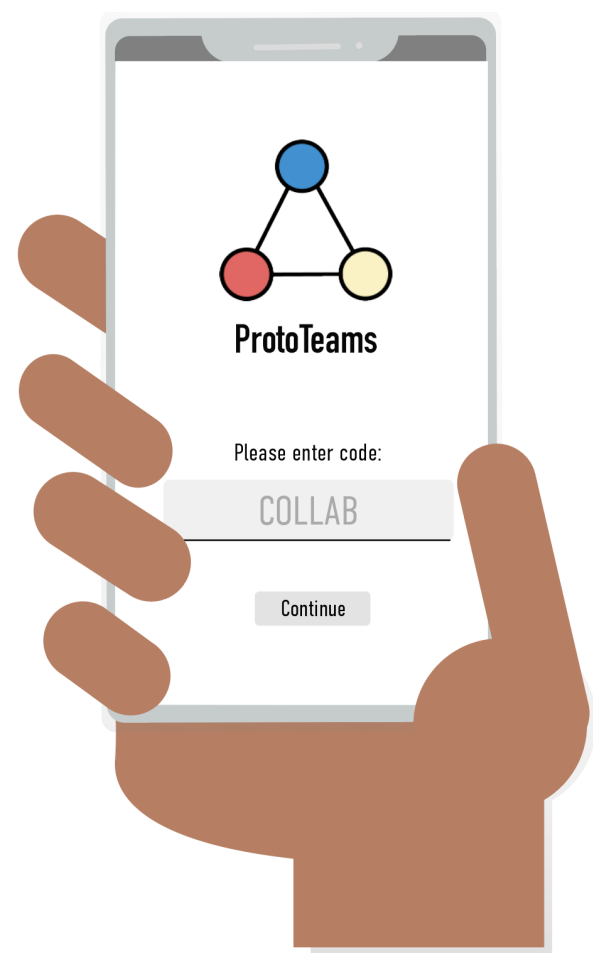

Figure 1: ProtoTeams leverages mobile devices to divide large co-located crowds into small groups.

\title{
ProtoTeams: Supporting Small Group Interactions in Co-Located Crowds
}

\section{Gustavo Umbelino}

University of California, San Diego

La Jolla, California

gumbelin@ucsd.edu

\section{Samuel Blake}

University of California, San Diego

La Jolla, California

sblake@ucsd.edu

\section{Amy Luo}

Carnegie Mellon University

Pittsburgh, Pennsylvania

awluo@andrew.cmu.edu

\author{
Vivian Ta \\ University of California, San Diego \\ La Jolla, California \\ vta@ucsd.edu
}

\section{Eric Truong}

University of California, San Diego

La Jolla, California

ehtruong@ucsd.edu

\section{Steven Dow}

University of California, San Diego

La Jolla, California

spdow@ucsd.edu

\section{ABSTRACT}

Group facilitators often organize large co-located crowds into small groups where people can more effectively interact and learn from each other. However, current approaches for facilitating small group interactions typically do not capture data about the rapport and future potential of interacting groups. We introduce ProtoTeams, a system designed to facilitate and gather data from small group interactions by dynamically connecting people from larger co-located crowds, such as classrooms and hackathons, through personal mobile devices. The system randomly assigns subjects into groups, then

Permission to make digital or hard copies of part or all of this work for personal or classroom use is granted without fee provided that copies are not made or distributed for profit or commercial advantage and that copies bear this notice and the full citation on the first page. Copyrights for third-party components of this work must be honored. For all other uses, contact the owner/author(s)

CSCW '19 Companion, November 9-13, 2019, Austin, TX, USA

(C) 2019 Copyright held by the owner/author(s).

ACM ISBN 978-1-4503-6692-2/19/11.

https://doi.org/10.1145/3311957.3359505 


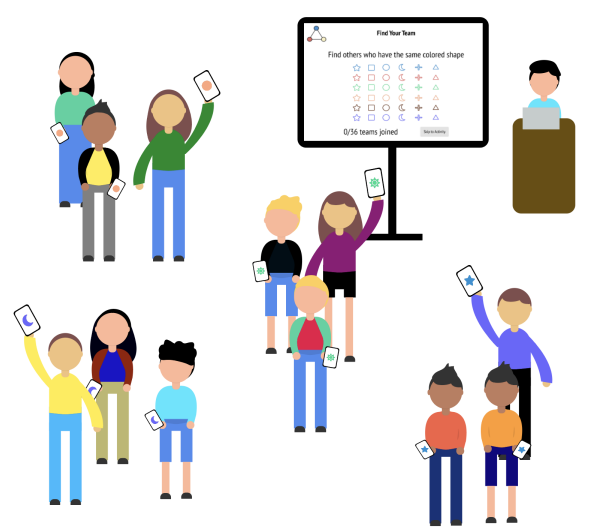

Figure 2: Participants hold their mobile devices up to find their assigned group members in the crowd. Shared display shows progress status. guides them through short activities, at the end of which they evaluate their teammate preferences. We report preliminary results from three deployments in a university-level design studio course where students $(N=50)$ interact with peers on activities before forming teams for a quarter-long project. Results suggest that the system is intuitive and groups form efficiently. Few subjects report the environment being too hectic and activities too short to accurately evaluate their peers. Future studies will continue investigating the value of this tool in co-located team dynamics.

\section{KEYWORDS}

co-located collaboration; team formation systems; team dating

\section{INTRODUCTION AND RELATED WORK}

Small group interactions are essential in everyday life and have been widely studied in multiple fields for decades. With the recent emphasis on teamwork [14], human-computer interaction $(\mathrm{HCl})$ researchers have developed systems and algorithms to aid different aspects of team dynamics, such as formation [1, 2, 4], collaboration [13], feedback exchange [9], communication [16], etc. Many of these systems aim to improve team performance and satisfaction. Nevertheless, there is ongoing debate on what criteria is important for selecting teammates, ideal team sizes, or the relative effectiveness between different team formation algorithms [12].

While there is great value in continuing research on these aspects of team dynamics, it is also important to note the intrinsic value of short, low-stakes interactions among individuals of a group. Small group interactions not only help individuals learn from each other [3], but may also help develop interpersonal relationships, which are important for collaborative work [6, 7].

Previous literature on team dating explores the formation of well-working teams without prior data on participants. Studies suggest that preference data generated after brief interactions between group members can help inform future team performance $[5,10,11]$.

In learning settings, balancing the needs of stakeholders (instructors and students) has also been shown to be an important factor in the design of team formation tools [8]. While students value transparency and agency over the criteria chosen to form teams, instructors value the efficiency of tool-based approaches. Previous systems have been designed to account for different stakeholder needs. On the instructor side, tools have been created to reduce the time and effort in managing teamwork in the classroom [17]. On the student side, tools that promote students' agency have been implemented [6]. There have also been systems that adopt a dynamic approach to team formation by coordinating rotation between remote team members [15].

This paper contributes to the literature of $\mathrm{HCl}$ systems by providing our experience designing, building, and testing ProtoTeams, a dynamic group assembly tool created to facilitate and collect data from face-to-face, small group interactions. 


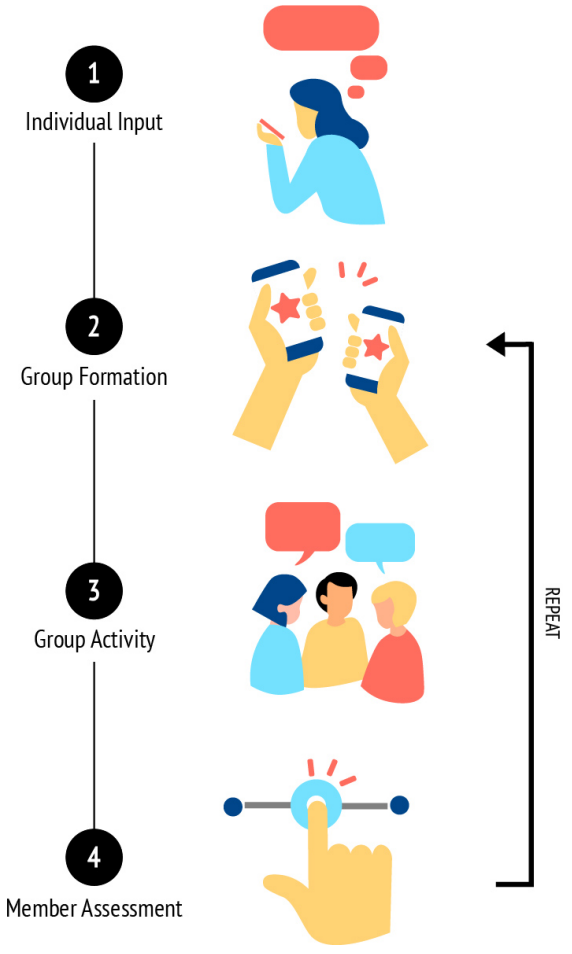

Figure 3: Activities flow diagram

Each activity we designed followed the same structure: (1) individual input, (2) group formation, (3) group activity, (4) member assessment. The type of activity and format of member assessment varied.

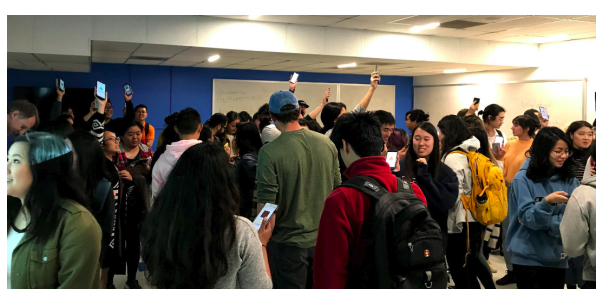

Figure 4: Participants using ProtoTeams to look for group members during group formation phase.

\section{SYSTEM DESIGN}

Inspired by "speed dating", the goal of ProtoTeams is to help individuals find potential teammates to interact with before committing to longer-term teams.

The system is subdivided into three distinct components: participant screen, shared display, and dashboard. The participant screen is where users interact with the system by providing input or following personalized instructions. The shared display provides an overview of the current activity stage by presenting key pieces of the system's status to both the host and participants, such as remaining time, session code, and formed groups (Figure 2). Finally, the dashboard is where the host can create sessions and customize different parameters of activities, such as duration, group sizes, and activity prompts.

As shown on Figure 3, each activity is composed of four stages: (1) users have a set amount of time to provide individual input into the system. (2) Individuals are assigned a colored shape and instructed to find others with the same icon (Figure 4). Participants find their assigned group members and provide confirmation to the system via the participant screen. (3) Once groups are confirmed, users participate in a timed group activity. (4) Finally, participants individually assess their interaction with each of their group members.

To inform the system's design for future work, we conducted three user testing sessions in a classroom setting. Our initial design decisions were made to satisfy the needs of the course.

Session 1: Icebreaker. The first session was composed of three icebreaker activities. The goal of this session was to form interpersonal relationships between classmates $(N=67)$. There was a total of 66 distinct groups. For the assessment phase, users were provided an interface with the following question: "Which group member would you see yourself working with again?" and buttons with each group member's name. To force conversion, only one name was allowed to be selected.

Session 2: Multiple-choice quiz (MCQ). This session was composed of three MCQ activities and its goal was to promote peer instruction. There was a total of $N=61$ participants and one round of group formation per quiz question (57 distinct groups). Based on interview feedback, in addition to each group member's name, we added an "ALL" option to the assessment interface. Similarly to Session 1 , only one option was allowed to be selected.

Session 3: Free-response quiz (FRQ). On this final session, the quiz was composed of two multiple choice questions and two free-response. Based on feedback from the previous session, we combined all the questions into one activity. As a result, there was only one round of group formation. For this activity, there was a total of $N=50$ subjects and 16 distinct groups. For the assessment stage, since "ALL" was a popular choice in the previous session, we updated the prompt to "Rate how likely you are 


\section{Table 1: FRQ results}

\begin{tabular}{|l|cc|cc|}
\hline & \multicolumn{2}{|c|}{$M C$ questions } & \multicolumn{2}{c|}{ FR questions } \\
\cline { 4 - 5 } \cline { 4 - 5 } & Correct & Incorrect & Serious & Non-serious \\
\hline \hline Individual & $72.3 \%$ & $27.7 \%$ & $94.6 \%$ & $5.4 \%$ \\
\hline Group & $80 \%$ & $20 \%$ & $100 \%$ & $0 \%$ \\
\hline
\end{tabular}

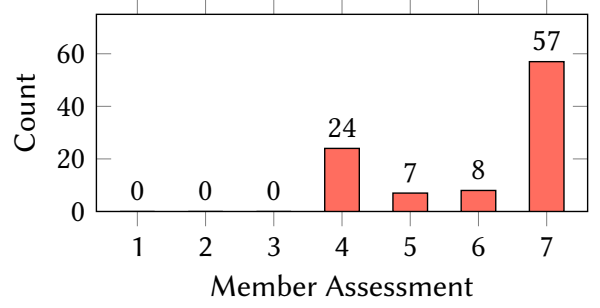

Figure 5: Likert scale member assessment distribution to work with these members again:" and redesigned the interface as a 7-point Likert scale per group member. The default value was set to 4 , "neutral", for each member.

\section{EVALUATION AND FUTURE WORK}

Different behavior patterns emerged from the crowd while interacting with ProtoTeams. In this section we report these emergent behaviors and potential implications for the future design of the system.

From video data recorded in testing sessions, we noticed that about half the class would hold their phone screens above their heads to better display their icons during the team formation stage. The other half held their phones lowered while looking at others' screens. A few students stood in one place with their screens out, waiting for others to find them. The majority of students actively searched for teammates around the room, and nobody seemed disengaged.

Once groups formed, most students remained in their teams near the center awaiting further instruction. This behavior obstructed remaining students who had to navigate around other groups to find their group members. It took about a minute for most groups to locate each other.

For the group input stage during the FRQ activity, group performance improved in comparison to individual performance both in terms of correctness and seriousness, shown by Table 1. Examples of non-serious responses are "Idk" or emojis. Causes for this improvement might be of interest for future experimental study.

People reported positively about their group members. The distribution for member assessments on a 7-point Likert scale is shown by Figure 5 .

From post-activity interviews, we received helpful feedback to guide improvements. Most comments were about the team formation stage. Participants felt that the process of finding groups was disordered as one subject mentioned, "the process seemed a bit hectic" and "everyone clustered in the center". For future iterations, it might be useful to instruct confirmed groups to relocate and stand towards the edge of the room to prevent clustering in the center. In addition, some people felt that the interactions were not long enough or meaningful enough. One subject noted that, "because it was super quick, everyone just kind of cut straight to the point". To address this issue, the time for the activities can be increased to encourage more in-depth discussion.

Although we have only deployed the system in a classroom setting, the possible design space is broad. Group activities are context-independent and thus can be easily adapted. Future iterations of this work will focus on deploying ProtoTeams in different settings, such as hackathons and civic meetings.

\section{REFERENCES}

[1] Waleed M. Al-Adrousy, Hesham A. Ali, and Taher T. Hamza. 2015. A Recommender System for Team Formation in MANET. J. King Saud Univ. Comput. Inf. Sci. 27, 2 (April 2015), 147-159. https://doi.org/10.1016/j.jksuci.2014.06.014 
[2] I. Amarasinghe, D. Hernrández-Leo, and A. Jonsson. 2017. Intelligent Group Formation in Computer Supported Collaborative Learning Scripts. In 2017 IEEE 17th International Conference on Advanced Learning Technologies (ICALT). 201-203. https://doi.org/10.1109/ICALT.2017.62

[3] Albert Bandura and Richard H Walters. 1977. Social learning theory. Vol. 1. Prentice-hall Englewood Cliffs, NJ.

[4] Rosanna Costaguta and María de los Angeles Menini. 2014. An Assistant Agent for Group Formation in CSCL Based on Student Learning Styles. In Proceedings of the 7th Euro American Conference on Telematics and Information Systems (EATIS '14). ACM, New York, NY, USA, Article 24, 4 pages. https://doi.org/10.1145/2590651.2590674

[5] Petru L. Curşeu, Patrick Kenis, Jörg Raab, and Ulrik Brandes. 2010. Composing Effective Teams through Team Dating. Organization Studies 31, 7 (2010), 873-894. https://doi.org/10.1177/0170840610373195

[6] Diego Gómez-Zará, Matthew Paras, Marlon Twyman, Jacqueline N. Lane, Leslie A. DeChurch, and Noshir S. Contractor. 2019. Who Would You Like to Work With? In Proceedings of the 2019 CHI Conference on Human Factors in Computing Systems (CHI '19). ACM, New York, NY, USA, Article 659, 15 pages. https://doi.org/10.1145/3290605.3300889

[7] Pamela J Hinds, Kathleen M Carley, David Krackhardt, and Doug Wholey. 2000. Choosing Work Group Members Balancing Similarity, Competence, and Familiarity. Organizational Behavior and Human Decision Processes 81, 2 (2000), 226 - 251. https://doi.org/10.1006/obhd.1999.2875

[8] Farnaz Jahanbakhsh, Wai-Tat Fu, Karrie Karahalios, Darko Marinov, and Brian Bailey. 2017. You Want Me to Work with Who?: Stakeholder Perceptions of Automated Team Formation in Project-based Courses. In Proceedings of the 2017 CHI Conference on Human Factors in Computing Systems (CHI '17). ACM, New York, NY, USA, 3201-3212. https: //doi.org/10.1145/3025453.3026011

[9] Markus Krause, Tom Garncarz, Jiaoliao Song, Elizabeth M. Gerber, Brian P. Bailey, and Steven P. Dow. 2017. Critique Style Guide: Improving Crowdsourced Design Feedback with a Natural Language Model. In Proceedings of the 2017 CHI Conference on Human Factors in Computing Systems (CHI '17). ACM, New York, NY, USA, 4627-4639. https: //doi.org/10.1145/3025453.3025883

10] loanna Lykourentzou, Robert E. Kraut, and Steven P. Dow. 2017. Team Dating Leads to Better Online Ad Hoc Collaborations. In Proceedings of the 2017 ACM Conference on Computer Supported Cooperative Work and Social Computing (CSCW '17). ACM, New York, NY, USA, 2330-2343. https://doi.org/10.1145/2998181.2998322

[11] Ioanna Lykourentzou, Shannon Wang, Robert E. Kraut, and Steven P. Dow. 2016. Team Dating: A Self-Organized Team Formation Strategy for Collaborative Crowdsourcing. In Proceedings of the 2016 CHI Conference Extended Abstracts on Human Factors in Computing Systems (CHI EA '16). ACM, New York, NY, USA, 1243-1249. https://doi.org/10.1145/2851581. 2892421

[12] Chinasa Odo, Judith Masthoff, and Nigel Beacham. 2019. Group Formation for Collaborative Learning. In Artificial Intelligence in Education, Seiji Isotani, Eva Millán, Amy Ogan, Peter Hastings, Bruce McLaren, and Rose Luckin (Eds.). Springer International Publishing, Cham, 206-212.

[13] Lora Oehlberg, Jasmine Jones, Alice Agogino, and Björn Hartmann. 2012. Dazzle: Supporting Framing in Co-located Design Teams Through Remote Collaboration Tool. In Proceedings of the ACM 2012 Conference on Computer Supported Cooperative Work Companion (CSCW '12). ACM, New York, NY, USA, 183-186. https://doi.org/10.1145/2141512.2141573

[14] Marcel M. Robles. 2012. Executive Perceptions of the Top 10 Soft Skills Needed in Today's Workplace. Business Communication Quarterly 75, 4 (2012), 453-465. https://doi.org/10.1177/1080569912460400

[15] Niloufar Salehi and Michael S. Bernstein. 2018. Hive: Collective Design Through Network Rotation. Proc. ACM Hum.Comput. Interact. 2, CSCW, Article 151 (Nov. 2018), 26 pages. https://doi.org/10.1145/3274420

[16] Amy X. Zhang and Justin Cranshaw. 2018. Making Sense of Group Chat Through Collaborative Tagging and Summarization. Proc. ACM Hum.-Comput. Interact. 2, CSCW, Article 196 (Nov. 2018), 27 pages. https://doi.org/10.1145/3274465 
[17] Haining Zhu, Nitish K. Rai, and John M. Carroll. 2018. SmartGroup: A Tool-Based Approach to Scale-free Small-group Learning Activities. In Companion of the 2018 ACM Conference on Computer Supported Cooperative Work and Social Computing (CSCW '18). ACM, New York, NY, USA, 261-264. https://doi.org/10.1145/3272973.3274070 